\title{
SMB operation for three-fraction separations \\ Purification of plasmid DNA
}

\section{Conference Paper}

Author(s):

Paredes, Galatea; Mazzotti, Marco; Stadler, Joachim; Makart, Stefan; Morbidelli, Massimo

Publication date:

2005

Permanent link:

https://doi.org/10.3929/ethz-b-000031203

Rights / license:

In Copyright - Non-Commercial Use Permitted

Originally published in:

Adsorption 11(S1), https://doi.org/10.1007/s10450-005-6033-1 


\title{
SMB Operation for Three-Fraction Separations: Purification of Plasmid DNA
}

\author{
GALATEA PAREDES AND MARCO MAZZOTTI* \\ ETH Swiss Federal Institute of Technology Zurich, Sonneggstrasse 3, CH-8092 Zurich, Switzerland \\ mazzohi@ivuk.mavt.ethz.ch \\ JOACHIM STADLER \\ Amersham Biosciences Europe GmbH, D-79111 Freiburg, Germany \\ STEFAN MAKART AND MASSIMO MORBIDELLI \\ ETH Swiss Federal Institute of Technology Zurich, Sonneggstrasse 3, CH-8092 Zurich, Switzerland
}

\begin{abstract}
The Simulated Moving Bed technology is extended to incorporate a cleaning in place step, and it is then applied by exploiting size exclusion chromatography to purify plasmid DNA. Experimental performances are discussed in the light of our theoretical understanding of the SMB behavior.
\end{abstract}

Keywords: simulated moving bed, plasmid DNA, size exclusion chromatography, cleaning in place

\section{Introduction}

After becoming an established technique for the separation of enantiomers of chiral compounds (Juza et al., 2000), the next challenge for the Simulated Moving Bed (SMB) technology is its application to bioseparations (Imamoglu, 2002). Examples of products that are considered for SMB separation are therapeutical proteins, antibodies, nucleosides and plasmid DNA. As in the case of chiral applications, it will be necessary to prove that SMB outperforms column chromatography and that the media used as stationary phases are stable enough. In this context an important issue is that of the regeneration of the media that is necessary in bio-separations and is called cleaning in place (CIP). This can be carried out by periodically contacting the column with a concentrated sodium hydroxide (1 $\mathrm{M}$ in this work) solution with the aim of removing all species that might have poisoned the media.

\footnotetext{
${ }^{*}$ To whom correspondence should be addressed
}

In this work, we present the application of the SMB process to one of the purification steps in the downstream processing of plasmid DNA (pDNA). There are a few novel results in this work. First, this is to the best of our knowledge the first application of SMB to pDNA purification. The separation principle is size exclusion, thanks to the wide differences in molecular size between pDNA and the other species in the feed mixture. Secondly, this is a group separation, where there is a large number of unspecified species in the feed all having different retention time in the columns due to different degrees of pore accessibility and retention. We extend the SMB design criteria to deal with this new situation. Finally, we utilize a SMB operation that has been introduced for three fraction separations, and is ideally suited for an on-line implementation of CIP.

\section{Interest of pDNA Purification}

Prokaryotic cells, e.g. bacteria, have one single chromosome containing its genetic information. This is a 
double stranded DNA molecule called chromosomic DNA or cDNA, which is circular in most of the cases. Many bacteria have in addition one or several extrachromosomal genetic elements, called plasmids, that are not essential, but confer an advantage to the cell. The plasmids have autonomous replication capacity and every specific plasmid has a characteristic average number of copies per cell, going from a few in some cases to several hundreds in others. A plasmid can be genetically modified in such a way that an interesting gene be inserted into it. A gene is a sequence of nucleotides (primary structural units of DNA and RNA) that codifies the necessary information to produce a certain protein, e.g., one having therapeutical interest. This possibility is at the basis of the plasmidbased therapies, where the plasmid is the so-called vector. It conveys the gene of interest to the cells and tissues of the patient, where its expression produces the required protein, exactly where it is needed (Ferreira et al., 2000).

The success of pDNA in gene therapy and for a new generation of vaccines depends on the development of safe and competitive production processes (Ferreira et al., 2000; Levy et al., 2000; Shamlou, 2003; Stadler et al., 2004). First, in a fermentation reactor the bacterial cells are grown. In order to harvest the plasmid that is produced in the cells, a lysis is carried out, to destroy the wall and cellular membrane. Lysis yields a mixture of cell debris (fragments of wall, membrane and other solids) that can be separated by centrifugation, thus obtaining the clarified lysate from which plasmid is to be purified. If the lysis is successful, most of the cDNA is separated with the cell debris during the centrifugation. It is required that the purified plasmid remain in the supercoiled circular covalently closed form (ccc pDNA). Even in the case of high copy number pDNA, its concentration in the clarified lysate does not exceed $3 \%$, whereas the other species are cDNA, RNA, proteins and endotoxins (Stadler et al., 2004). The latter are liposaccharides (LPS), which are abundant in the outer cell membrane of most bacteria and are released in large amounts during lysis. Endotoxins are toxic and therefore their removal is absolutely necessary.

The separation principle investigated in this work is the size exclusion mechanism, which is a conventional way of separating pDNA from RNA in preparative chromatography (Stadler et al., 2004). Plasmid DNA is excluded from the pores of the media, whereas RNA, proteins, salts, and other small molecules are eluted later. Endotoxins and cDNA are larger than pDNA in principle, but are fragmented during lysis and sample preparation and therefore are only partly excluded from the pores.

\section{Experimental}

Nine chromatographic columns $(10 \mathrm{~cm}$ length, $1 \mathrm{~cm}$ internal diameter, $V=7.85 \mathrm{ml}$ ) were packed with the size exclusion media Sepharose 6 Fast Flow from Amersham Biosciences (90 $\mu \mathrm{m}$ particle size). Eight columns were used in the SMB, while the ninth was used for analytical purposes. The packing quality and column efficiency were assessed through acetone and potassium chloride injections. A modified ÄKTAexplorer unit (Amersham Biosciences) was used to run the SMB experiments, as well as the analysis (Abel et al., 2003). All experiments were performed in a $100 \mathrm{mM}$ Tris/HCl buffer with $10 \mathrm{mM}$ EDTA and 2 M ammonium sulfate (AS) (Stadler et al., 2004). All chemicals were purchased from Fluka (Buchs, Switzerland). Three different pDNA samples were used, ranging from 5 to 8 kilobases. The first (pDNA sample 1) was obtained from Strathmann Biotec AG (Dengelsberg, Germany), whereas the second (pDNA sample 2) and the third (pDNA sample 3) were prepared in our labs during two independent lysis of frozen cell obtained from the same fermentation batch. The clear lysate was concentrated before SMB separation using an ultrafiltration unit from Amersham Biosciences, which is equipped with a hollow fibre membrane with a $300 \mathrm{kDa}$ NMWC (nominal molecular weight cutoff) operated at a flux of $20 \mathrm{l} /\left(\mathrm{hm}^{2}\right)$. Samples from SMB product streams were analyzed after desalting by gel filtration.

\section{Design of the CIP-SMB Separation}

The separation of pDNA from RNA is carried out in the CIP-SMB unit, whose scheme is illustrated in Fig. 1. It is constituted of five sections (column configuration 1-2-2-2-1), which are labelled $0,1,2,3$, and $r$. Sections 1 to 3 constitute a standard three section SMB where the species excluded from the pores are separated from those having access to the pores, and are collected in the raffinate. CIP takes place in section 0 , using a proper regeneration solvent, namely a $1 \mathrm{M}$ $\mathrm{NaOH}$ solution. Section $\mathrm{r}$ is required to re-equilibrate the column that has undergone CIP with the solvent 


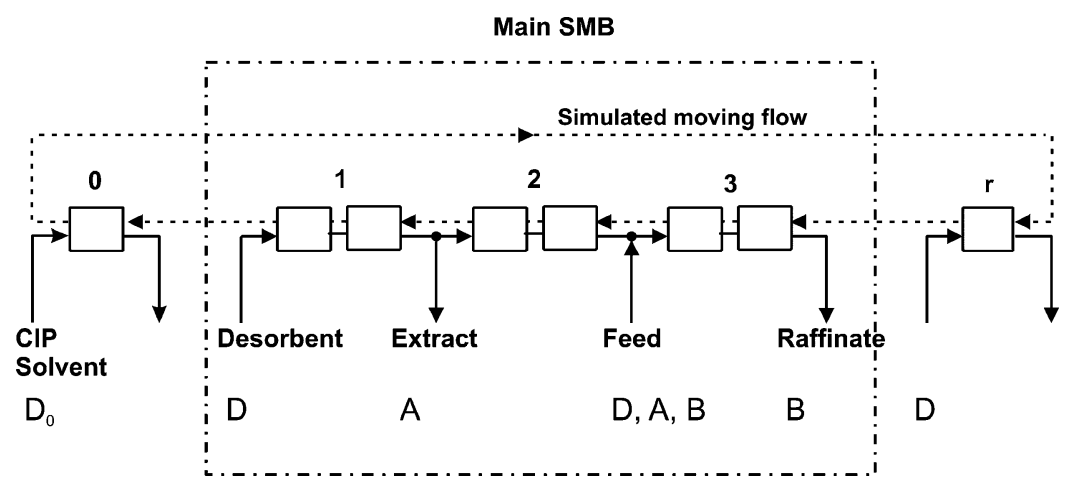

Figure 1. Scheme of a CIP-SMB unit for the separation of the more retained species A and the less retained species B.

buffer system used for the separation. This is a special case of the three fraction SMB operation that has been recently demonstrated through experiments (Abel et al., 2003) and simulations (Paredes et al., 2004). A similar scheme was proposed also in the patent literature (Voigt et al., 2001).

The SMB operating conditions are conveniently given in terms of flow rate ratios, $m_{j}$, that are defined in each section of the unit as follows (Mazzotti et al., 1997; Migliorini et al., 1999):

$$
m_{j}=\frac{Q_{j} t^{*}-V \varepsilon-V_{j}^{D}}{V(1-\varepsilon)}
$$

where $V$ and $\varepsilon$ are bed volume and void fraction, respectively, and $Q_{j}$ and $V_{j}^{D}$ are the flow rate and the extra-column dead volume (per column) in section $j$, respectively (through potassium chloride pulse injec-

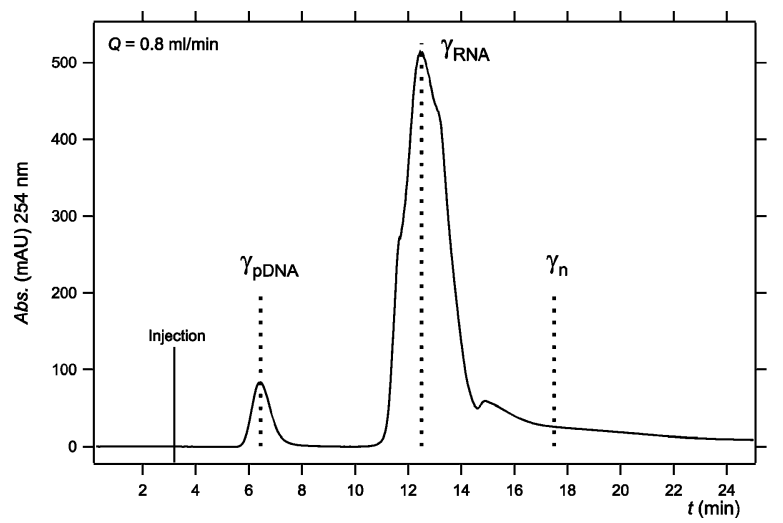

tions the following values in $\mathrm{ml}$ were obtained: $V_{0}^{D}=$ $0.015 ; V_{1}^{D}=0.073 ; V_{2}^{D}=0.135 ; V_{3}^{D}=0.068$; $\left.V_{r}^{D}=0.015\right)$.

In order to determine the range of feasible $m_{j}$ values a number of single column experiments have been carried out, and the two most significant ones are shown in Fig. 2, namely a pulse chromatogram of the feed mixture and a complete CIP/regeneration cycle. The retention times of the chromatographic peaks in Fig. 2(left), as well as the breakthrough times of the $\mathrm{pH}$ fronts in Fig. 2(right), are:

$$
t_{i}^{R}=V\left(\varepsilon+(1-\varepsilon) \gamma_{i}\right) / Q
$$

where the parameter $\gamma_{i}$ depends on the fraction of pores accessible to species $i, \varepsilon_{i}^{p}$, and on retention according to the equation $\gamma_{i}=\varepsilon_{i}^{p}+\left(1-\varepsilon_{i}^{p}\right) K_{i}$, with $K_{i}$ accounting for its possible retention (it coincides with the Henry's

Figure 2. (left) Chromatogram of a $500 \mu \mathrm{L}$ injection of pDNA sample 2 at $Q=0.8 \mathrm{ml} / \mathrm{min}$; the retention times selected to calculate $\gamma_{i}$ in Eq. (2) are highlighted; injection is performed at 3.2 minutes. (right) Chromatogram illustrating saturation of a column with the CIP solution $(1 \mathrm{M} \mathrm{NaOH})$ and regeneration with the buffer solution $(Q=0.9 \mathrm{ml} / \mathrm{min})$. 
constant for linear adsorption). We evaluate the experiments in Fig. 2 as follows: by assuming that pDNA is completely excluded from the pores and elutes with the first peak, i.e. $\gamma_{\mathrm{pDNA}}=0$ and $\varepsilon=0.32$; by observing that the second peak in Fig. 2(left) is broad and constituted of a group of species, and by describing it through a characteristic time for the elution of the first, big peak, i.e. $\gamma_{\mathrm{RNA}}=0.92$ in Fig. 2(left), and a characteristic time for the elution of the tail, i.e. $\gamma_{n}$, which is between 1.6 and 2.0 depending on the batch (the value of $\gamma_{\text {RNA }}$ indicates pore accessibility and some interaction with the resin, probably due to the high ammonium sulfate concentration; $\gamma_{n}$ should be viewed just as an important parameter for the design of section 1 of the SMB-see Eq. (4)); by noticing that the times where the $\mathrm{pH}$ of the CIP solution is reached and where regeneration is complete are very similar, and can be characterized by $\gamma_{r}=1.7$.

Using these values, and following the design approach presented earlier (Paredes et al., 2004), the following conditions for complete separation and proper regeneration in the CIP-SMB operation of Fig. 1 can be written:

$$
\begin{aligned}
\gamma_{r} & \leq m_{0} \\
\gamma_{n} & \leq m_{1} \\
\gamma_{\mathrm{pDNA}} & \leq m_{2} \leq \gamma_{\mathrm{RNA}} \\
\gamma_{\mathrm{pDNA}} & \leq m_{3} \leq \gamma_{\mathrm{RNA}} \\
\gamma_{r} & \leq m_{r}
\end{aligned}
$$

It is worth noting that conditions (3) and (7) apply only where the same buffer and the same CIP solution as in the experiment of Fig. 2(right) are used. Moreover, these do not consider the need for a specified contact time with the CIP solution that might be a requirement by drug regulatory authorities and will be considered in a successive work.

\section{Experimental Results, Discussion, and Conclusions}

Four SMB experiments were carried out at the conditions reported in Table 1, where also the raffinate quality and the productivity are reported. The latter parameter is calculated as the ratio between feed flow rate and SMB volume, i.e. $8 \mathrm{~V}$, multiplied by the pDNA concentration in the feed. Since this concentration is difficult to quantify and depends on the sample, a reference value of $1 \mathrm{mg} / \mathrm{ml}$ has been used in all runs for comparison. In runs $\mathrm{A}$ to $\mathrm{C}$ isocratic conditions were adopted, hence sections 0 and $r$ were used only for testing of the experimental set-up; the values of $m_{0}$ and $m_{r}$ in run $\mathrm{C}$ are in fact so small in order to save solvent. On the contrary in run D the CIP-SMB operation was implemented, by using the CIP solution in section 0 and by carrying out regeneration in section $r$. Since this was a proof-of-concept run, $m_{0}$ and $m_{r}$ values much larger than the theoretical limit have been adopted. In all runs the cyclic steady state was reached after two to three cycles.

It can be readily observed that all runs fulfill the separation requirements of Eqs. (4) to (6), and in fact in runs A to $\mathrm{C}$ no pDNA could be detected in the extract, and no other species but pDNA could be found in the raffinate. This is illustrated with an example in Fig. 3(left) where the analysis of the raffinate produced during run $\mathrm{C}$ is shown. One can observe the pDNA peak, but no peak for the non-excluded species. The negative peak on the right is assumed to be a system peak (solvent peak) due to the difference in composition between the analytical mobile phase and the solvent in the injected pulse. It is worth noting that runs A and B have been carried under the same conditions but with samples from different batches.

Run D has been successfully carried out in the CIPSMB mode. This has been carefully verified by checking that the $\mathrm{pH}$ at the outlet of the column in section $r$ reached the value corresponding to the mobile phase

Table 1. Operating conditions and separation performance of the experimental runs; the indication pure raffinate means that the concentration of the impurity was below the limits of detection.

\begin{tabular}{lccccccccc}
\hline Run & Sample & $t^{*}[\mathrm{~min}]$ & $m_{0}$ & $m_{1}$ & $m_{2}$ & $m_{3}$ & $m_{r}$ & Raff. quality & $\operatorname{Pr}[\mathrm{mg} /(\mathrm{h} \mathrm{ml})]$ \\
\hline A & 1 & 5 & 2.71 & 2.94 & 0.439 & 0.762 & 0.651 & Pure & 0.316 \\
B & 2 & 5 & 2.71 & 2.94 & 0.439 & 0.762 & 0.651 & Pure & 0.316 \\
C & 2 & 5 & 0.651 & 2.44 & 0.271 & 0.762 & -0.005 & Pure & 0.487 \\
D & 3 & 5 & 3.12 & 2.19 & 0.271 & 1.01 & 4.02 & Impurities & 0.744 \\
\hline
\end{tabular}



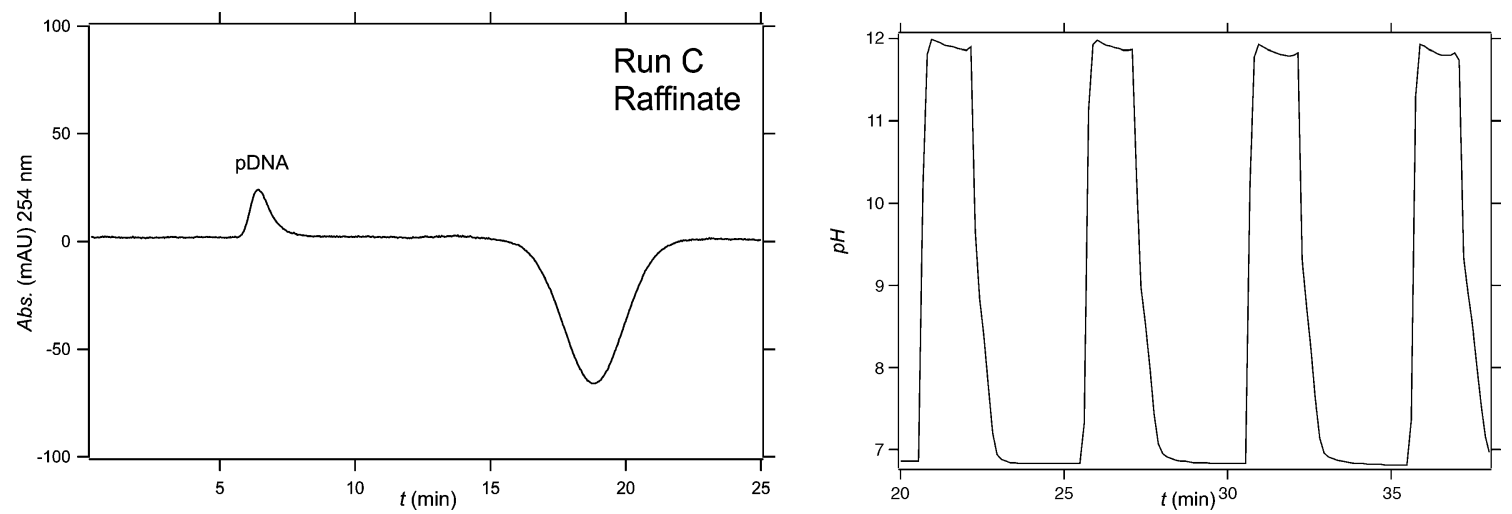

Figure 3. (left) Run C: Chromatogram of a $500 \mu \mathrm{L}$ injection of the raffinate ( $Q=0.8 \mathrm{ml} / \mathrm{min})$. (right) Run D: $\mathrm{pH}$ profile at the outlet of the re-equilibration section during four switch periods at cyclic steady state.

buffer used in sections 1 to 3 of the SMB. That this is indeed the case is illustrated in Fig. 3(right) where the $\mathrm{pH}$ profile at the outlet of section $\mathrm{r}$ is monitored for a few switch periods. Since the chosen value of $m_{r}$ is much larger than its lower bound, namely 4.02 instead of 1.7 , actually complete regeneration of the column is achieved much earlier than necessary, as expected. In run $\mathrm{D} m_{3}$ is larger than its upper bound in Eq. (6), thus leading to a larger productivity but also to the presence of some pollutants in the raffinate, which are evident in the gel electrophoresis. This is typical of separation processes, particulary of SMB, where the best compromise between purity and productivity has to be found.

These results indicate that the CIP-SMB configuration proposed can indeed be used for the purification of bio-molecules, particularly plasmid DNA. Further work will address the potential improvement of performance with SMB as compared to column chromatography, and the possible use of CIP-SMB in a way that complies with the regulations of the drug administration bodies.

\section{Acknowledgments}

The support of the Swiss Innovation Promotion Agency (KTI, project number 6138.1 KTS) is gratefully acknowledged. We thank Prof. Sven Panke of ETHZurich for useful discussions and support.

\section{References}

Abel, S., M. Bäbler, C. Arpagaus, M. Mazzotti, and J. Stadler, "TwoFraction and Three-Fraction Continuous SMB Separation of Nucleosides," J. Chromatogr. A, 1043, 201-210 (2004)

Ferreira, G.N.M., G.A. Monteiro, D.M.F. Prazeres, and J.M.S. Cabral, 2000, "Review: Downstream Processing of Plasmid DNA for Gene Therapy and DNA Vaccine Applications," Tibtech 18, 380-388, (200).

Imamoglu, S., "Simulated Moving Bed Chromatography (SMB) for Application in Bioseparation," Advances in Biochemical EngineeringBiotechnology, 76, 211-231 (2002).

Juza, M., M. Mazzotti, and M. Morbidelli, "Simulated Moving-Bed Chromatography and its Application to Chirotechnology," Trends in Biotechnology, 18, 108-118 (2000).

Levy, S.M., R.D. OḰennedy, A.P. Shamlou, and P. Dunnill, "Review: Biochemical Engineering Approaches to the Challenges of Producing Pure Plasmid DNA,” Tibtech, 18, 296-305 (2000).

Mazzotti, M., G. Storti, and M. Morbidelli, "Optimal Operation of Simulated Moving Bed Units for Nonlinear Chromatographic Separations," J. Chromatogr. A, 769, 3-24 (1997).

Migliorini, C., M. Mazzotti, and M. Morbidelli, "Simulated Moving Bed Units with Extracolumn Dead Volume," AIChE J., 45, 14111422 (1999).

Paredes, G., S. Abel, M. Mazzotti, M. Morbidelli, and J. Stadler, Ind. Eng. Chem. Res., in press (2004).

Shamlou, P. A., "Review: Scaleable Processes for the Manufacture of Therapeutic Quantities of Plasmid DNA," Biotechnol. Appl. Biochem., 37, 207-218 ( 2003).

Stadler, J., R. Lemmens, and T. Nyhammar, "Review: Plasmid DNA Purification," Journal gene medicine, 6, S54-S66 (2004).

Voigt, U., J. Kinkel, R. Hempel, and R. M. Nicoud, "Chromatographic Process for Obtaining Highly Purified Cyclosporin A and Related Cyclosporins," United States Patent No.: US006306306B1, 2001. 